\title{
Research on Rapid Assessment of Earthquake Death Toll Based on Neural Network
}

\author{
Haoyu $\mathrm{Wu}^{1,2}$, Xinyan $\mathrm{Wu}^{3 *}$, Hongwei $\mathrm{Li}^{2}$, Yong $\mathrm{Luo}^{2}$ \\ ${ }^{1}$ University of Science and Technology of Taiyuan, Taiyuan, Shanxi 030024, China \\ ${ }^{2}$ Earthquake Administration of Shanxi Province, Taiyuan, Shanxi 030021, China \\ ${ }^{3}$ Institute of Geophysics, China Earthquake Administration, Beijing 100081, China

\section{基于神经网络的地震死亡人数快速评估研究 ${ }^{1}$ \\ 吴昊昱 ${ }^{1,2}$, 吴新燕 $3^{*}$, 李宏伟 ${ }^{2}$, 罗勇 ${ }^{2}$ \\ ${ }^{1}$ 太原理工大学, 山西太原 030024 \\ ${ }^{2}$ 山西省地震局, 山西太原 030021 \\ ${ }^{3}$ 中国地震局地球物理研究所, 北京 100081}

\begin{abstract}
101 post-1960 fatal earthquakes in China mainland were selected to build a BP neural network model for rapid earthquake fatality estimation. In this model, event time, magnitude, epicenter intensity, population density and the event region were selected as input vectors. While, the fatality was assigned as output vector. An empirical test shows that the BP neural network model can give a more accurate assessment, with a good match to the real death toll. This approach can be applied in rapid earthquake losses estimation, and provide scientific basis for emergency rescue and government decision-making.
\end{abstract}

Keywords: death toll; BP neural network; earthquakes; rapid assessment

\section{摘要}

选取了 1960 年以来的 101 个中国大陆地区造成人 员死亡的地震震例, 选取地震发生的时间、震级、 震中烈度、震中人口密度和地震所在地区作为影响 地震人员伤亡的主要因素建立输入层神经元, 地震

1 基金项目 2015 年度地球物理研究所基本科研业务费 (DQJB15C09)

*作者简介 吴吴昱 (1980 -), 男, 工程师, 主要从事数 字地震学与震害评估方面的工作, E-mail:

why5489@aliyun.com

通信作者 吴新燕 (1977-), 女, 高级工程师, 主要从事 防震减灾与灾害评估方面的工作, E-mail: wuxy1977@sina.com
死亡人数作为输出层神经元, 利用 $\mathrm{BP}$ 神经网络为 平台, 建立了地震死亡人数快速评估模型。实证检 验表明神经网络模型快速评估的地震死亡人数更 接近真实情况, 评估结果更精确。神经网络地震死 亡人数快速评估模型可以应用到地震灾害快速评 估, 为政府决策和救援提供参考依据。

关键词: 死亡人数; $\mathrm{BP}$ 神经网络; 地震; 快速评 估

1. 引言

突发的破坏性地震会在瞬间给人类社会造成 巨大的损失, 包括建筑物和生命线工程损坏、人员 伤亡以及经济损失等。人员伤亡指标是评判地震灾 害程度的主要指标, 研究地震人员伤亡的评估方法 对于减少地震损失具有重要的意义。因此越来越多 的中外学者开始关注地震生命损失的研究 [1-7], 尤其是 2008 年汶川地震的发生, 更加快了相关研 究的步伐。这些评估方法各有特色, 使用的影响因 素、统计资料和评估角度也各不相同, 结果预测与 实际情形都或多或少的存在差距。本文试图利用神 经网络理论, 考虑多个因素对地震伤亡的影响,

借以更加全面的建立评估模型。当下神经网络 研究已经变成一个科学热点, 涉及多个领域如火灾 防护领域 ${ }^{[8]}$, 交通领域 ${ }^{[9-10]}$, 医学领域 ${ }^{[1]}$, 经济领 域 ${ }^{[12]}$ 等, 尤其在地震科学领域应用非常广泛, 例如 地震预测 ${ }^{[13-18]}$, 灾害评估 ${ }^{[19-20]}$, 地震序列判别 ${ }^{[21-22]}$ 以及危险性划分 ${ }^{[23]}$ 等几个方面。 
Risk Analysis and Crisis Response in Big Data Era (RAC-16)

\section{2. 人工神经网络的原理}

人工神经网络(Artificial Neural Networks, ANN) 受动物的神经系统启发, 逼近或从样本中学习目标 值为离散值、实值或者向量的函数提供一个实用而 一般的方法。正如动物神经系统的构成, 人工神经 网络也是由许多个神经元相互连接组织形成一个 网络。

本文研究的是地震发生后的快速死亡人数评 估模型, 选取地震发生的时间, 震级, 震中烈度, 人口密度, 以及地震区域作为评估地震伤亡的 5 个 基本参数, 因为这 5 个参数随时间变化范围较小, 在短时期内可以看作是稳定值, 便于模型的快速评 估。

根据杨杰英等[24]的研究, 地震发生时间是影 响伤亡人数的重要因素：夜晚比白天的地震伤亡 大，尤其是在 00-06 点期间。而震级与震中烈度更 是描述地震能量释放大小与建筑物破坏的两个物 理量, 两个物理量与地震人员的伤亡均为正相关。 人口密度也与伤亡人数呈正相关, 地震发生时, 震 中在经济发达人口稠密区比震中在人口稀少或者 无人区造成的经济损失与人员伤亡更大。不同区域 的发生同样级别的地震造成的人员伤亡截然不同, 这跟当地人们的防灾意识与当地房屋的抗震性能 都息息相关。建立图 1 三层神经网络模型, 5 个基 本参数作为输入层, 地震死亡人数作为输出层。

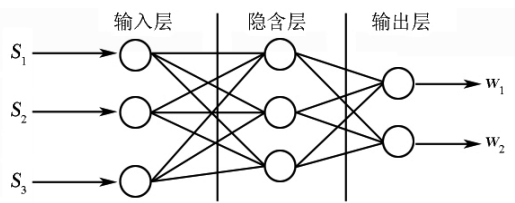

图 13 层 BP 神经网络结构示意图[20]

Fig 1 Sketch diagram of three layers BP neural network

\section{3. 地震样本数据收集}

选取了 1959 年至 2013 年近 55 年来中国大陆 地区造成人员死亡的地震共 160 个，剔除一些 “极 端震例” 如 1975 年的辽宁海城地震和 1976 年的四 川松潘平武地震都因为有一定的预防措施减少了 人员的地震死亡数量; 还有震中在海洋和无人区造 成死亡的震例和死亡人数为 1 人的震例, 这些都是 为了保证评估值更接近一般情况下的地震生命损 失情况。最终保留了 101 个震例作为样本 (见表 1)。 为了方便计算机进行仿真拟合，地震发生在白天 (8:00 20:00) 用 1 表示, 夜晚（20:00 次日 8:00） 用 2 表示; 根据我国的行政区划图和地震烈度区划 图将我国分为东，中，西三个区域 (见表 2)，其中 东部区域用 1 表示, 中部区域用 2 表示, 西部区域 用 3 表示。

表 1 震例表

Table 1 the earthquake cases

\begin{tabular}{|c|c|c|c|c|c|c|c|}
\hline 序号 & 地点 & $\begin{array}{l}\text { 发震 } \\
\text { 时间 }\end{array}$ & 震级（M） & $\begin{array}{l}\text { 震中 } \\
\text { 烈度 }\end{array}$ & $\begin{array}{c}\text { 人口密度(人 } \\
\text { / } \mathrm{km} 2 \text { ) }\end{array}$ & 区域 & $\begin{array}{l}\text { 死亡 } \\
\text { 人数 }\end{array}$ \\
\hline 1 & 河北唐山 & 2 & 7.8 & 11.0 & 11000.00 & 1 & 242000 \\
\hline 2 & 四川汶川 & 1 & 8.0 & 11.0 & 103.41 & 3 & 69227 \\
\hline 3 & 云南通海 & 2 & 7.7 & 10.0 & 71.34 & 3 & 15621 \\
\hline ! & ! & ! & : & i & ! & ! & : \\
\hline 100 & 云南盐津 & 2 & 5.1 & 6.0 & 93.76 & 3 & 2 \\
\hline 101 & 重庆荣昌 & 1 & 5.3 & 7.0 & 735.29 & 2 & 2 \\
\hline
\end{tabular}

表 2 分区表

Table 2 division districts

\begin{tabular}{c|c}
\hline 区域 & 省份 \\
\hline 东部 & $\vdots$ 黑龙江、吉林、辽宁、北京, 天津, 河北, 山东, 江苏, 上海, 浙江, 福建, 广东, 海南, 广西 \\
中部 & 内蒙古, 山西, 陕西, 宁夏, 甘肃, 重庆, 河南, 湖北, 安徽, 江西, 湖南, 贵州 \\
西部 & 青海, 西藏, 新疆, 云南, 四川 \\
\hline
\end{tabular}


Risk Analysis and Crisis Response in Big Data Era (RAC-16)

\section{4. 评估模型分析}

在 matlab 平台上建立 BP 神经网络模型, 输入 层为 5 个参数, 输出层为地震死亡人数, 利用隐含 层 25 个神经元个数, 学习速度 0.05 , 训练 30000 次。

通过平台程序, 得到 BP 网络训练结果如图 2 图 4 所示, 可以看出网络训练所得的误差很小。图 5 是实际伤亡人数与网络输出值之间训练和测试的 对比图, 显然二者之间非常接近, 误差较小, 因此, 可以用 $\mathrm{BP}$ 神经网络对地震死亡人数进行预测评 估。

\section{5. 算例}

根据 2014 年中国发生的四川康定 6.3 级地震和 云南鲁甸发生的 6.5 级地震的震例 (见表 3) 对 BP 神经网络地震死亡人数预测评估进行检验, 并与经 验拟合的多项式评估算法[6-7], 结果 (见表 4) 表 明: BP 神经网络模型在评估大地震造成人员死亡

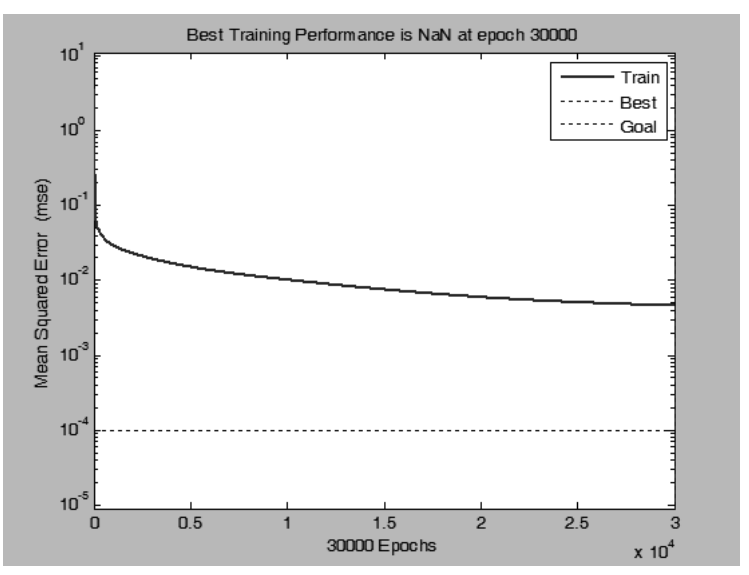

图 2 训练性能

Fig 2 Training performance

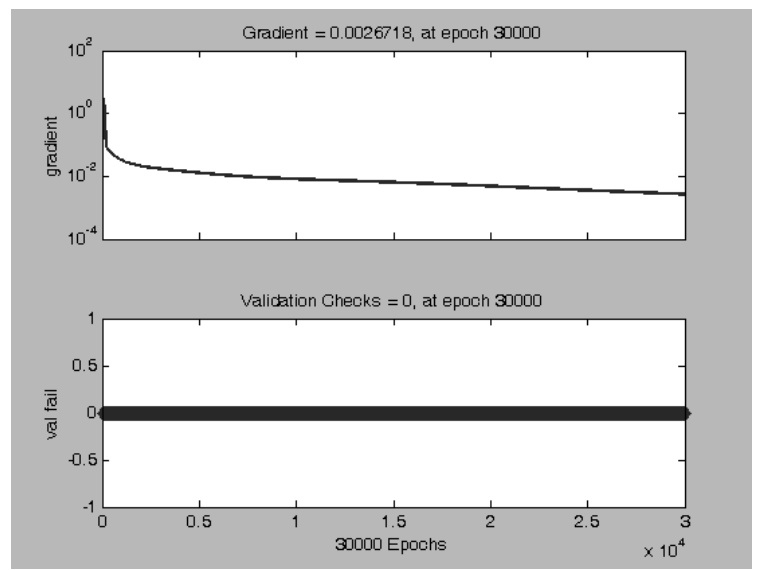

图 3 训练状态

Fig 3 Training state 
Risk Analysis and Crisis Response in Big Data Era (RAC-16)

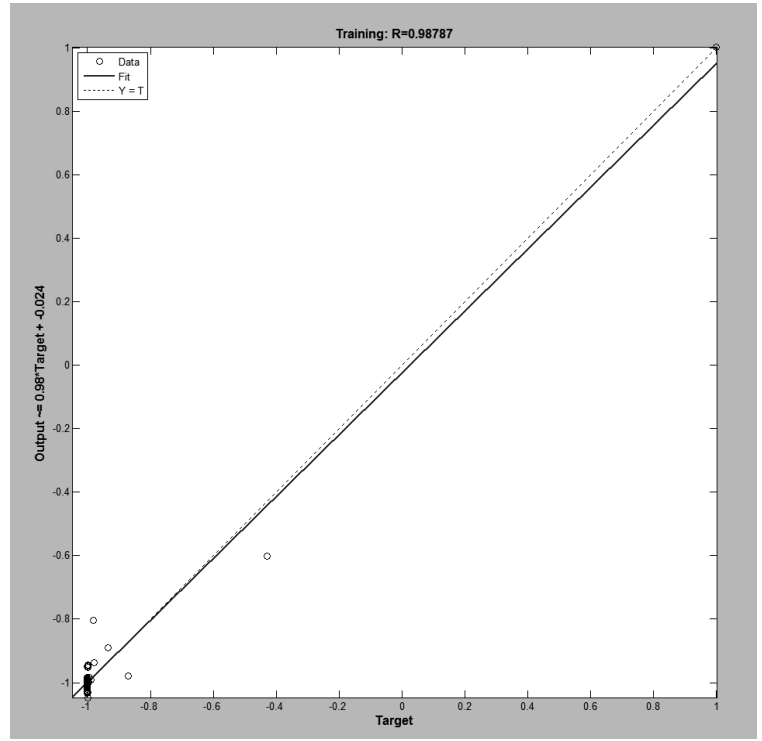

图 4 回归结果

Fig 4 regression result

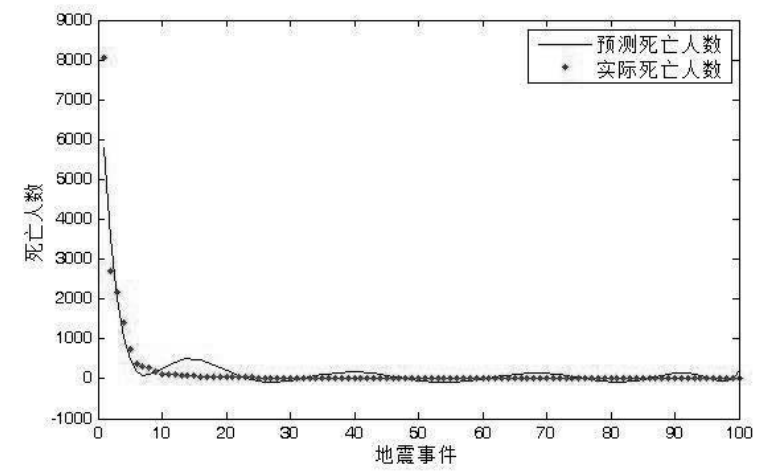

图 5 实际样本值与预测值对比图

Fig 5 Contrast between actual sample values and predicted values

人数较多的地震时, 结果更加精确, 误差率仅为 $16.21 \%$ 而其他方法建立的模型，误差率较大，而在 评估死亡人员数量较小的地震时, 误差大些, 但评 估数量在同一数量级, 考虑到数量较少的人员死亡 有一定的偶然性和随机性, 因此综合评估, BP 神 经网络的误差率更小, 结果更精确。
6. 结论

分析了造成人员伤亡的几个主要因素利用 BP 神经网络的神经元构建了地震死亡人数评估模型, 此预测方法, 比以往的经验算法的精度更高。

1975 年的海城 M7.3 地震与 1976 年的松潘-平 武 M7.2 地震因为之前都有预报措施, 因此这两次 
Risk Analysis and Crisis Response in Big Data Era (RAC-16)

表 3 地震算例各参数值

Table 3 parameter value of the earthquake examples

\begin{tabular}{|c|c|c|c|c|c|c|}
\hline 地点 & 时间 & $\begin{array}{l}\text { 震级 } \\
(M)\end{array}$ & 震中烈度 & $\begin{array}{l}\text { 人口密度 } \\
\left(\text { 人 } / \mathrm{km}^{2}\right)\end{array}$ & 区域 & 死亡人数 \\
\hline 四川康定 & 1（白天） & 6.3 & 8 & 9.57 & 3 (西部) & 5 \\
\hline 云南鲁甸 & 1（白天） & 6.5 & 9 & 265 & 3 (西部) & 617 \\
\hline
\end{tabular}

表 4 评估结果对比

Table 4 Comparison of evaluation results

\begin{tabular}{cccccccc}
\hline \multirow{2}{*}{ 震例 } & 实际死 & \multicolumn{3}{c}{ 肖光先 ${ }^{[6]}$} & \multicolumn{2}{c}{ 赵洪声 ${ }^{[7]}$} & \multicolumn{2}{c}{ BP 神经网络 } \\
\cline { 3 - 7 } & 亡人数 & 预测值 & 误差率 $\%$ & 预测值 & 误差率 $\%$ & 预测值 & 误差率\% \\
\hline 康定地震 & 5 & 0 & 100 & 2 & 60 & 1 & 80 \\
鲁甸地震 & 617 & 209 & 66.12 & 146 & 76.34 & 517 & 16.21 \\
\hline
\end{tabular}

地震的死亡人数分别是 1328 人和 41 人。用 $\mathrm{BP}$ 神 经网络进行模拟估算, 如果这两次地震没有震前预 报措施, 死亡的人数将会是 12765 人和 1204 人, 分别减少了 $79 \%$ 和 $96 \%$ 的人员死亡, 由此可见地震 前有效的预报措施可大量的减少地震造成的人员 伤亡。

结果表明: 传统的经验拟合算法，一般只考虑 1-3 个参数作为地震死亡人数的因素, 而且误差较 大, 而 BP 神经网络评估模型则考虑了更多因素, 充分显示了地震造成人员死亡的多样性和复杂性, 并且拟合误差较小, 评估预测值更加接近真实情 况。当然因为影响地震死亡人数的因素还有很多, 对各影响因素之间的权重评估还需要建立更客观, 更科学和更合理的评估体系, 还需要做进一步的研 究与探讨。

\section{参考文献}

[1] 马玉宏, 谢礼立. 我国社会可接受地震人员死 亡率的研究. 自然灾害学报, 2001.10(3):56-63.

[2]马玉宏,谢礼立. 地震人员伤亡估算方法研究. 地 震工程与工程振动, 2000, 20(4):140-147.

[3] 赵振东,郑向远. 地震人员伤亡研究的回顾与 进展.自然灾害学报, 2000, 9(1):93-99.

[4] Kishor Jaiswal, Wald, et al. Estimating casualties for large earthquakes worldwide Using an Empirical Approach, USGS Open-file Report, 2009

[5] K.S. Jaiswal D.J. Wald P.S. Earle K.A. Porter and M. Hearne. Earthquake Casualty Models within the USGS Prompt Assessment of Globa Earthquakes for Response (Pager) System. Second International Workshop on Disaster Casualties, 15-16 June 2009.

[6] 高惠瑛, 李清霞.地震人员伤亡快速评估模型
研究.灾害学,2010,25: 275-277.

[7] 高建国,贾艳.地震救援能力的一项指标: 地震 灾害发布时间的研究.灾害学, 2005, 20(1): 31-35.

[8] W Zhu, Q You. High-rise Building Group Regional Fire Risk Assessment Model Based on AHP. Journal of Risk Analysis and Crisis Response, 2016, 6(1):31-37.

[9] 乔维德.遗传算法和神经网络在交通事故预测 中的应用.电气传动自动化, 2008,30(1):41-44.

[10] 刘秀清,宇仁德,范东凯. 基于广义回归神经网 络的交通事故预测. 山东理工大学学报 (自然科 学版), 2007,21(2):28-31.

[11] 张诣. 混沌神经网络算法研究及其在医学图像 处理中的应用.大连理工大学.2013.

[12] 王建成,高大启等. 改进的遣传和 BP 杂交算法 及神经网络经济预警系统设计.系统工程理论 与实践, 1998, 18(4):136-141.

[13] 冯利华. 基于神经网络的地震活动性研究. 西 北地震学报, 2000.22(4):402 -406.

[14] 王碧泉. 模式识别研究及其在地震学中的应用. 地球物理学报, 1994,37 卷增刊 I: 215-223.

[15] 陈一超, 曾三友, 张好春等. 基于遗传神经网络 的地震预测研究. 计算机应用与软件, 2008,25(4) : 135-137.

[16] 温岩,乔兵. 基于 MATLAB 工具箱的神经网络 在地震预报中的应用.东北地质研究, 2001,(3): 38- 39.

[17] 王炜,宋先月. 人工神经网络在地震中短期预 报中的应用.中国地震, 2000.16(2):149- 157.

[18] 魏红梅,黄世源,贺曼秋. 基于 MATLAB 工具箱 的地震预测模型. 东北地震研究, 2007(3):64-68

[19] 石成钢,刘西拉. 人工神经元网络在震害预测 中的应用。地震工程与工程振动, 1991.11(2):39-47.

[20] 钱枫林,崔健. BP 神经网络模型在应急需求预 测中的应用-—以地震伤亡人数预测为例. 中 
Risk Analysis and Crisis Response in Big Data Era (RAC-16)

国安全科学学报, 2013.23(4): 20-25.

[21] 王炜,戴维乐. 使用 Mf 值、C 值、D 值异常资 料进行地震短期预测的神经网络方法. 中国地 震, 1997.13(4):392- 401.

[22] 李冬梅. 基于人工神经网络和模式识别方法的 地震序列类型早期预测研究 $[\mathrm{D}]$. 中国地震局兰 州地震研究所.2012.

[23] 李强, 徐戈, 杨彩霞. 人工神经网络在潜在地震
危险区估计中的应用.华北地震科学, 2007. 25(2):36- 41 .

[24] 杨杰英,李永强,刘丽芳等. 地震三要素对地震 伤亡人数的影响分析. 地震研究, 2007. 30(2):182-187. 